\title{
Identification of the OGLE Blazars behind the Large and Small Magellanic Clouds
}

\author{
Natalia Żywucka ${ }^{1, *}$, Arti Goyal ${ }^{1}$, Marek Jamrozy ${ }^{1}$, Łukasz Stawarz $^{1}$, Michał Ostrowski ${ }^{1}$, \\ Szymon Kozłowski ${ }^{2}$ and Andrzej Udalski ${ }^{2}$ \\ 1 Astronomical Observatory, Jagiellonian University, ul. Orla 171, 30-244 Kraków, Poland; \\ arti@oa.uj.edu.pl (A.G.); jamrozy@oa.uj.edu.pl (M.J.); stawarz@oa.uj.edu.pl (Ł.S.); mio@oa.uj.edu.pl (M.O.) \\ 2 Warsaw University Observatory, Al. Ujazdowskie 4, 00-478 Warszawa, Poland; \\ simkoz@astrouw.edu.pl (S.K.); udalski@astrouw.edu.pl (A.U.) \\ * Correspondence: n.zywucka@oa.uj.edu.pl
}

Received: 30 October 2017; Accepted: 22 November 2017; Published: 28 November 2017

\begin{abstract}
We report the selection of blazar candidates behind the Large and Small Magellanic Clouds. Both flat spectrum radio quasar and BL Lacreate objects were selected based on the long-term, multi-colour Optical Gravitational Lensing Experiment photometric data. We cross-correlated the Magellanic Quasar Survey catalogue of spectroscopically confirmed quasars and quasar candidates located behind the Magellanic Clouds with the radio data at six frequencies from 0.8 to $20 \mathrm{GHz}$. Among the 1654 objects visible in optical range, we identified a sample of 44 newly selected blazar candidates, including 27 flat spectrum radio quasars and $17 \mathrm{BL}$ Lacs. We examined selected objects with respect to their radio, optical, and mid-infrared properties.
\end{abstract}

Keywords: galaxies: active; flat spectrum radio quasars and BL Lacertae blazars; individual: Small and Large Magellanic Clouds

\section{Introduction}

The Optical Gravitational Lensing Experiment (OGLE) has observed a few million stars in the area of both Magellanic Clouds (MCs). The Magellanic Quasars Survey (MQS) [1] is based on an OGLE-III phase of the experiment and was designed to increase the number of the identified active galactic nuclei (AGN) behind both MCs. This survey covers 100\% of the LMC and $70 \%$ of the SMC OGLE-III areas, i.e., $42 \mathrm{deg}^{2}$. The AGN candidates found in the MC fields were analysed in a four-step selection process: mid-infrared properties, optical variability, ROSAT X-ray counterparts, and, finally, optical spectroscopy study. Eventually, the MQS catalogue contains 758 quasars; around $10-15 \%$ of them should be radio loud [2] and several of them may be the flat spectrum radio quasar (FSRQ) type blazars according to the AGN unification scheme [3]. During the selection process of the MQS quasars, some of the AGN candidates were rejected in the spectroscopic analysis due to the lack of particular emission lines in their optical spectra and were listed in the separate list of featureless sources (FS), although still obeying any of the mid-infrared and optical variability criteria. The FS list consists of 669 and 229 objects from the LMC and the SMC fields, respectively, and some of them may be blazars of the BL Lac type.

Below, we present the selection procedure of blazars among the OGLE-detected AGN and AGN candidates. In particular, we cross-matched the MQS catalogue and the FS list with four radio catalogues, the Sydney University Molonglo Sky Survey (SUMSS) [4] at $0.843 \mathrm{GHz}$, the Australia Telescope $20 \mathrm{GHz}$ (AT20G) [5] at 5, 8, and $20 \mathrm{GHz}$, the Parkes-MIT-NRAO (PMN) [6] at $4.85 \mathrm{GHz}$, and the Australia Telescope PMN follow-up (ATPMN) [7] at 4.8 and $8.6 \mathrm{GHz}$, to disclose both types of blazars. 


\section{Methodology}

The blazar candidates were selected based on the MQS catalogue and the FS list via (i) the radio and optical positioning; (ii) the cross-matching procedure; and (iii) the parameters examination of identified objects.

The positions of the selected quasars from the MQS catalogue and the sources from the FS list were examined against the positions of radio sources from the two radio catalogues: the SUMSS at $0.84 \mathrm{GHz}$ and the ATCA at 4.8 and $8.6 \mathrm{GHz}$.

The cross-matching procedure was based on the archival data from four sky surveys: the SUMSS, AT20G, ATPMN, and PMN. We searched for the radio counterparts by cross-matching the optical positions of the MQS and FS sources with the positions of radio sources listed in the radio catalogues, using the standard spherical trigonometric distance procedure. Since various radio and optical surveys have different resolutions, we chose an error circle within which a source can be identified to be coincident with the same source from different catalogues: if the computed radius of the error circle, $\Delta r$, was found to be less than the square root of the quadratic sum of $1 / 3 \mathrm{rd}$ of optical and radio resolutions ( $\sim 1^{\prime \prime}$ is assumed at optical frequencies), the radio counterpart was considered to be coincident with the optical source.

The further examination of the selected objects was based on the three parameters: the radio loudness $(R)$, the radio spectral index $\left(\alpha_{r}\right)$, and the mid-infrared spectral index $\left(\alpha_{I R}\right)$. OGLE I-band photometric observations and radio ATPMN, AT20G, or SUMSS data were used to calculate $R$, while $\alpha_{r}$ were obtained for all the sources observed at at least two different frequencies. Mid-infrared data were provided by the Infrared Array Camera (IRAC) and Multiband Imaging Photometer for SIRTF (MIPS) instruments onboard the Spitzer Space Telescope, at 3.6, 4.5, 5.8, and 8.0 microns.

Figure 1 shows the radio contours overlayed on the gray scale optical images for the two exemplary identified blazar candidates. We used the Digitized Sky Survey (DSS) R-band optical images as grey scales and the radio data at $8.6,4.85$ or $0.845 \mathrm{GHz}$ as contours. A black cross marks the optical position from the MQS catalogue or the FS list of each blazar candidate. All sources, which were found exclusively in the PMN catalogue, as well as other sources which were not associated with the peak of the radio emission, are considered as dubious and marked with the "a" symbol in the final list of the blazar candidates.
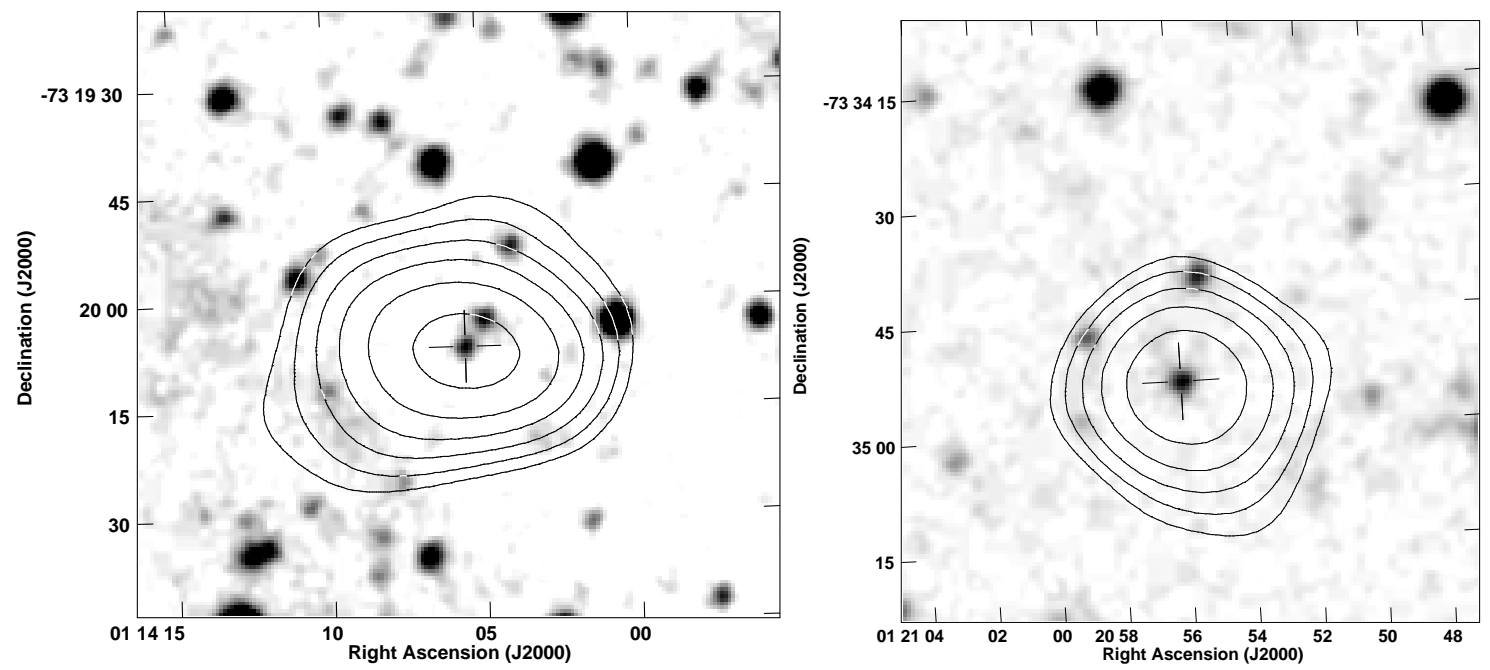

Figure 1. Exemplary contour maps of two identified blazar candidates, J0114-7320 and J0120-7334.

We searched for the fractional linear polarization degree $\left(\mathrm{PD}_{\mathrm{r}}\right)$ and linear polarization angle $\left(\mathrm{PA}_{\mathrm{r}}\right)$ of all the selected candidates to further verify if the selected objects are indeed blazars. We have examined the AT20G catalogue and analyzed polarized flux densities on 4.8 and $8.6 \mathrm{GHz}$ radio maps 
of the MCs. Finally, we extracted nine objects from the selected sample: six FSRQs and three BL Lacs (listed in Table 1), noting that all of them are strongly polarized radio sources with $\mathrm{PD}_{\mathrm{r}, 4.8 \mathrm{GHz}}>3 \%$.

Table 1. The degree of linear polarization and the polarization angle of the FSRQ and BL Lac blazar type candidates.

\begin{tabular}{|c|c|c|c|c|c|c|c|}
\hline \multirow{3}{*}{ Object } & \multicolumn{5}{|c|}{$\mathbf{P D}_{\mathbf{r}}$} & \multicolumn{2}{|c|}{$\mathbf{P A}_{\mathbf{r}}$} \\
\hline & $4.8 \mathrm{GHz}$ & $5 \mathrm{GHz}$ & $8 \mathrm{GHz}$ & $8.6 \mathrm{GHz}$ & $20 \mathrm{GHz}$ & $4.8 \mathrm{GHz}$ & $8.6 \mathrm{GHz}$ \\
\hline & $(\%)$ & $(\%)$ & $(\%)$ & $(\%)$ & $(\%)$ & $\left({ }^{\circ}\right)$ & $\left({ }^{\circ}\right)$ \\
\hline (1) & (2) & (3) & (4) & (5) & (6) & (7) & (8) \\
\hline \multicolumn{8}{|c|}{ FSRQ blazar type candidates } \\
\hline $\begin{array}{l}\text { J0114-7320 } \\
\text { J0120-7334 } \\
\text { J0442-6818 } \\
\text { J0512-6732 } \\
\text { J0551-6916 } \\
\text { J0551-6843 } \\
\end{array}$ & $\begin{array}{l}9.5 \pm 0.5 \\
5.0 \pm 0.3 \\
3.3 \pm 0.2 \\
7.3 \pm 0.4 \\
9.1 \pm 0.5\end{array}$ & $\begin{array}{l}11.7 \\
12.7\end{array}$ & $\begin{array}{l}10.0 \\
10.7\end{array}$ & $7.0 \pm 0.4$ & $\begin{array}{c}8.1 \\
13.6\end{array}$ & $\begin{array}{c}70.7 \pm 14.7 \\
-52.2 \pm 0.7 \\
13.6 \pm 2.1 \\
8.3 \pm 38.2 \\
-3.9 \pm 40.4\end{array}$ & $-12.9 \pm 14.3$ \\
\hline \multicolumn{8}{|c|}{ BL Lac blazar type candidates } \\
\hline $\begin{array}{c}\text { J0111-7302 } \\
\text { J0501-6653 } \\
\text { J0518-6755 a }\end{array}$ & $\begin{array}{c}4.1 \pm 0.2 \\
10.6 \pm 0.5 \\
12.6 \pm 0.6\end{array}$ & 8.3 & 8.3 & $22.7 \pm 1.1$ & 9.7 & $\begin{array}{c}4.7 \pm 34.1 \\
-2.8 \pm 25.6 \\
-16.6 \pm 11.0\end{array}$ & $-11.1 \pm 46.1$ \\
\hline
\end{tabular}

NOTE- ${ }^{a}$ Dubious object. Columns: (1) source designation, (2)-(6) linear polarization degree $\left(\mathrm{PD}_{\mathrm{r}}\right)$ at $4.8,5,8$, 8.6 , and $20 \mathrm{GHz},(7)$ and (8) linear polarization angle $\left(\mathrm{PA}_{\mathrm{r}}\right)$ at 4.8 and $8.6 \mathrm{GHz}$.

\section{Results}

The sky distribution of our blazar candidates sample is presented in Figure 2. The main results of blazar identification are listed below:

1. We define two samples of 44 blazar candidates including 27 FSRQs and 17 BL Lacs.

2. All objects in our sample are optically faint with the V band magnitude between 18 and 22 . All the FSRQ candidates are distant sources with redshifts from 0.286 up to 3.320. The redshift distribution for BL Lacs is unknown due to the lack of the emission lines in their optical spectra.

3. The $R$ parameter for the selected sources varies from 12 to $4.45 \times 10^{3}$ for the FSRQ candidates and from $1.71 \times 10^{2}$ to $7.02 \times 10^{3}$ for the BL Lac candidates meaning that all the candidates are radio-loud indeed.

4. The estimated values of $\alpha_{r}$ vary from -0.57 to 1.37 . Since we used the non-simultaneous archival radio data in the analysis, the $\alpha_{r}$ may indicate radio flux variability for the sampled sources, as it is expected for blazars.

5. The estimated values of $\alpha_{I R}$ are between -0.44 and 3.07. The infrared colours indicate that the infrared continua of the selected sources are non-thermal in origin, as expected for blazars.

6. The $\mathrm{PD}_{\mathrm{r}}$ and $\mathrm{PA}_{\mathrm{r}}$ at 5, 8, and $20 \mathrm{GHz}$ were taken from the AT20G catalogue and were measured by us from the archival 4.8 and $8.6 \mathrm{GHz}$ radio maps. All the blazar candidates for which we have found polarimetric data are strongly polarized sources with the average polarization of $\mathrm{PD}_{\mathrm{r}, 4.8} \sim 6.8 \%$ at $4.8 \mathrm{GHz}$. The 4.8 and $5 \mathrm{GHz}$ data were obtained with the same telescope but presumably on different dates. The fact that, for the two objects, i.e., J0512-6732 and J0111-7302, observed at both 4.8 and $5 \mathrm{GHz}$, there are very different $\mathrm{PD}_{\mathrm{r}}$ in these close radio bands, suggests polarization variability, which is a further indication that they are blazar like objects.

Considering the $\alpha_{I R}, R$, and $\mathrm{PD}_{\mathrm{r}}$ parameters together, we suggest that nine candidates, including six FSRQ and three BL Lac type objects, can be safely recognized as newly identified blazars. 

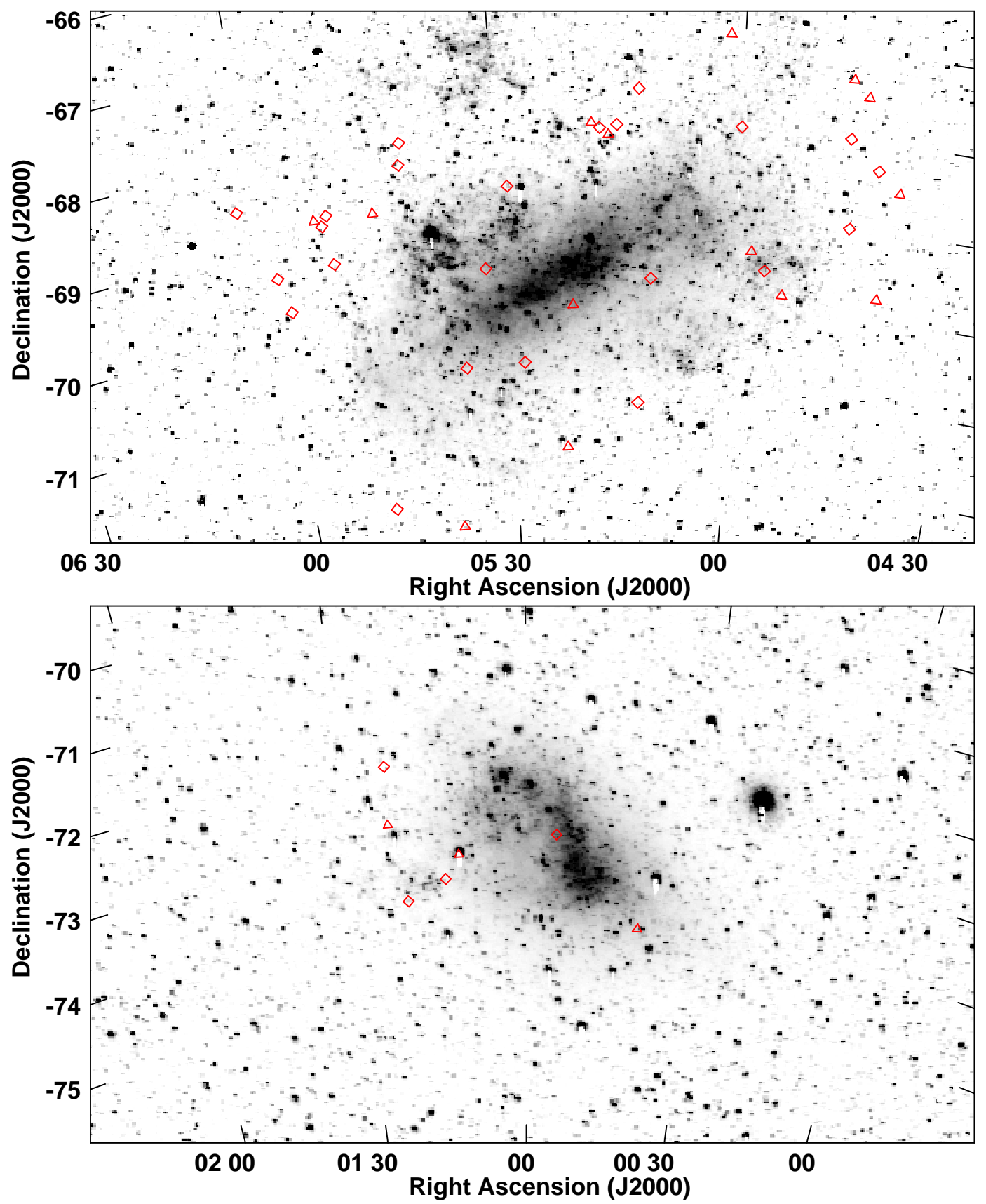

Figure 2. The distribution of the selected blazar candidates behind the Large (top panel) and the Small (bottom panel) MCs. The FSRQ candidates are shown with red open diamond symbols and BL Lac candidates are shown with red open triangular symbols. The gray scales of both MC optical images are taken from [8].

Acknowledgments: N.Ż. ackowledges support of the Polish National Science Centre (NCN) through the grant 2014/15/N/ST9/05171. A.G. and M.O. acknowledge support from the NCN through the grant 2012/04/A/ST9/00083. A.G. also acknowledges partial support from 2013/09/B/ST9/00026. Ł.S. was supported by the Polish NSC grant 2016/22/E/ST9/00061. The OGLE project has received funding from the NCN MAESTRO grant No. 2014/14/A/ST9/00121 to A.U. S.K. also acknowledges financial support from the NCN OPUS grant No. 2014/15/B/ST9/00093.

Author Contributions: S.K. and A.U. provide the optical data form the OGLE survey; N.Ż. conducted the data gathering, data analysis, correlations studies, and discussion; M.J. and A.G. were involved in data gathering and analysis; and Ł.S. and M.O. were involved in the interpretation of the obtained results. 
Conflicts of Interest: The authors declare no conflict of interest.

\section{Abbreviations}

The following abbreviations are used in this manuscript:

$\begin{array}{ll}\text { AGN } & \text { Active Galactic Nuclei } \\ \text { BL Lac } & \text { BL Lacertae } \\ \text { FS } & \text { Featureless Sources } \\ \text { FSRQ } & \text { Flat Spectrum Radio Quasar } \\ \text { LMC } & \text { Large Magellanic Cloud } \\ \text { MQS } & \text { Magellanic Quasars Survey } \\ \text { OGLE } & \text { Optical Gravitational Lensing Experiment } \\ \text { SMC } & \text { Small Magellanic Cloud }\end{array}$

\section{References}

1. Kozłowski, S.; Onken, C.A.; Kochanek, C.S.; Udalski, A.; Szymański, M.K.; Kubiak, M.; Pietrzyński, G.; Soszyński, I.; Wyrzykowski, Ł.; Ulaczyk, K.; et al. The Magellanic Quasars Survey. III. Spectroscopic Confirmation of 758 Active Galactic Nuclei behind the Magellanic Clouds. Astrophys. J. 2013, 775, 92.

2. Kellermann, K.I.; Sramek, R.; Schmidt, M.; Shaffer, D.B.; Green, R. VLA observations of objects in the Palomar Bright Quasar Survey. Astron. J. 1989, 98, 1195-1207.

3. Urry, C.M.; Padovani, P. Unified Schemes for radio-loud active galactic nuclei. Publ. Astron. Soc. Pac. 1995, 107, 803-845.

4. Bock, D.C.-J.; Large, M.I.; Sadler, E.M. SUMSS: A Wide-Field Radio Imaging Survey of the Southern Sky. I. Science Goals, Survey Design, and Instrumentation. Astron. J. 1999, 117, 1578-1593.

5. Murphy, T.; Sadler, E.M.; Ekers, R.D.; Massardi, M.; Hancock, P.J.; Mahony, E.; Ricci, R.; Burke-Spolaor, S.; Calabretta, M.; Chhetri, R.; et al. The Australia Telescope $20 \mathrm{GHz}$ Survey: The source catalogue. Mon. Not. R. Astron. Soc. 2010, 402, 2403-2423.

6. Condon, J.J.; Griffith, M.R.; Wright, A.E. The Parkes-MIT-NRAO surveys. IV-Maps for the Southern Survey covering delta in the range -88 to $-37 \mathrm{deg}$. Astron. J. 1993, 106, 1095-1100.

7. McConnell, D.; Sadler, E.M.; Murphy, T.; Ekers, R.D. ATPMN: Accurate positions and flux densities at 5 and $8 \mathrm{GHz}$ for 8385 sources from the PMN survey. Mon. Not. R. Astron. Soc. 2012, 422, 1527-1545.

8. Bothun, B.D.; Thompson, I.B. Observations with the parking lot camera. I-Surface photometry and color distribution of the Magellanic Clouds. Astrophys. J. 1988, 96, 877-883. 